\title{
Calculation of IR-spectra of structural fragments of lignins
}

\author{
O.Yu. Derkacheva*, and M.M. Ishankhodzhaeva \\ St. Petersburg State University of industrial technologies and design, 198095 St. Petersburg, Russia
}

\begin{abstract}
To study structure of softwood lignins the experimental and theoretical IR-spectra in middle IR-diapason were analyzed. To interpret these data the quantum chemical calculations of IR-spectra of general dimmer fragments of softwood lignins by method of density functional theory (DFT/B3LYP) with 6-31G(d,p) as basis set were carried out. These calculations showed that frequencies of normal vibrations of fragment with $\beta$-alkyl-aryl linkage are close to the experimental values of the IR absorption bands of lignin, and infrared spectrum of this structure is similar to the experimental spectrum of lignin. The calculations with accounting for the solvent showed a strong increase in the intensity of the majority of the bands and the solvent effect on the frequencies of vibrations.
\end{abstract}

Lignins, being part of the plant raw, are valuable source of chemical compounds [1]. It is why heightened interest to decoding native lignin structure is saved. Lignin is not individual substance. Lignin is mixture of irregular aromatic polymers of close structure. Therefore the structural formula of lignin does not exist.

At the same time it is known that lignins consist of phenylpropane units of guaiacyl, syringyl and p-coumaryl types [2]. Currently it is revealed more than 20 types of linkages between phenylpropane units. It is established that $\beta$-alkyl-aryl linkage is the most common one in native lignins.

One of useful methods of lignin analysis is FTIR-spectroscopy. By quantitative analysis of IR-spectra and construction of calibration dependencies similar "structure-properties" the tasks of finding the most informative absorbance bands to characterize chemical structure of native lignins are quite successfully solved [3-7]. In order to increase the reliability of the interpretation of IR spectra there is a necessity of the comparative analysis of the experimental and theoretical data. To achieve this aim in the work the quantum-chemical calculations of IR spectra of dimmer models of softwood lignin were carried out by method of density functional theory with the B3LYP functional in the basis of $6-31+\mathrm{G}(\mathrm{d}, \mathrm{p})$ using a software package Gaussian08. IR-spectra were calculated for two main structural elements of guaiacyl lignin.

Figure shows the lignin experimental spectrum and the simulated IR spectra of the investigated dimmers in the form of the absorption curves in the frequency range $700-$ $1900 \mathrm{~cm}^{-1}$. By comparison with experiment the frequencies of normal vibrations of the

\footnotetext{
*Corresponding author: derkachevaou@rambler.ru
} 
dimmers were multiplied by a scaling factor 0.975 for the frequency range $400-1900 \mathrm{~cm}^{-1}$ and 0.950 for the region $2700-4000 \mathrm{~cm}^{-1}$ [8-9].

The calculation results showed that the frequencies of normal vibrations of the dimmer with $\beta$-O-4 linkage between aromatic rings are close to the experimental values of the infrared absorption bands of lignin, and the calculated infrared spectrum (curve 2 a) is similar to the experimental spectrum of lignin (curve 1).

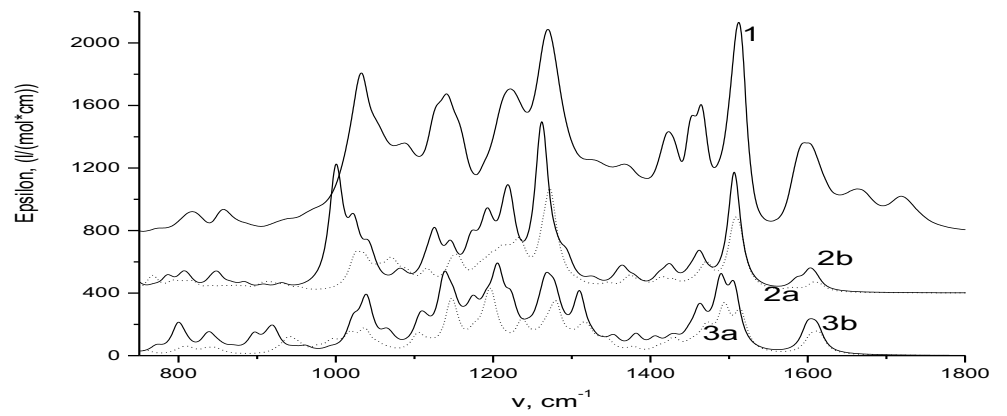

Fig. 1. IR-spectrum of softwood lignin (1) and molar absorption coefficient of dimmers with $\beta-O-4$ (2) and with $\alpha-\mathrm{O}-4$ и $\beta-5$ linkages (3) in vacuum (a) and in water (b)

IR-spectrum of model with $\alpha-\mathrm{O}-4$ and $\beta-5$ linkages between aromatic rings (curve $3 \mathrm{a}$ ) is markedly different from spectrum of the previous model. We can note, for example, the difference between the frequencies of in-plane skeletal vibration of two aromatic rings of the dimmer near $1500 \mathrm{~cm}^{-1}$, which is the cause of an appearance of doublet with frequencies at 1498 and $1517 \mathrm{~cm}^{-1}$. Quantum chemical calculations showed a marked increase in the intensities of the bands at 1150 and $1200 \mathrm{~cm}^{-1}$ and the appearance of strong bands at 1320 and $940 \mathrm{~cm}^{-1}$. According to calculations, these bands are associated with inplane vibrations of aromatic ring with four substitutes.

In the work an influence of environment on the spectra of both dimmers was considered. To see a solvent effect (water) the calculations were carried out by method of PCM (model of polarization continuum Tomasi). From Fig. 1 we can see that the IRspectra calculated by quantum-chemical method of PCM (curves $2 b$ and $3 b$ ) demonstrate an increasing intensity of dimmer absorbance bands as result of environment influence. One can also note shift of absorbance bands of aromatic rings on $7-10 \mathrm{~cm}^{-1}$ to lower frequencies.

Thus comparing theoretical IR-spectra of the studied structural fragments with experimental spectra of softwood lignins allows to clarify an empirical assignment of absorbance bands. Later it will allow to evaluate structure of lignins of different origin.

\section{References}

1. I. P. Deimeko, Khimija Rastitel'nogo Syr'ja 16, 5 (2012) (in Russian)

2. S.Y. Lin, C.W. Dence, Methods in lignin chemistry (Springer-Verlag, Berlin, 1992)

3. K.V. Sarkanen, H.M. Chang, G.G. Allan, Tappi J. 50, 587 (1967)

4. A.P. Karmanov, O.Yu. Derkacheva, Russ. J. Bioorganic Chem. 39, 677 (2013)

5. O.Yu. Derkacheva, Journal of Applied Spectroscopy 80, 670 ( 2013)

6. N.N. Chipanina et al., Chemistry of Natural Compounds 31, 392 (1995)

7. O. Faix, Holzforschung 40, 273 (1986)

8. A.P. Scott, L. Radom, J. Phys. Chem. 100, 16502 (1996)

9. S. Barsberg, Theor. Chem. Acc. 134, 33 (2015) 